\title{
Using RF Transmissions from loT Devices for Occupancy Detection and Activity Recognition
}

\author{
Wenda Li, Member, IEEE, Shelly Vishwakarma, Member, IEEE, Chong Tang, Student Member, IEEE, \\ Karl Woodbridge, Member, IEEE, R. J. Piechocki, Member, IEEE, Kevin Chetty Member, IEEE
}

\begin{abstract}
T ecosystems consist of a range of smart devices that generated a plethora of Radio Frequency (RF) transmissions. This provides an attractive opportunity to exploit already-existing signals for various sensing applications such as e-Healthcare, security and smart home. In this paper, we present Passive loT Radar (PloTR), a system that passively uses RF transmissions from loT devices for human monitoring. PloTR is designed based on passive radar technology, with a generic architecture to utilize various signal sources including the WiFi signal and wireless energy at the Industrial, Scientific and Medical (ISM) band. PloTR calculates the phase shifts caused by human motions and generates Doppler spectrogram as the representative. To verify the proposed concepts and test in a more realistic environment, we evaluate PloTR with four commercial loT devices for home use. Depending on the effective signal and power strength, PloTR performs two modes: coarse sensing and fine-grained sensing. Experimental results show that PloTR can achieve an average of $91 \%$ in occupancy detection (coarse sensing) and $91.3 \%$ in activity recognition (fine-grained sensing).
\end{abstract}

Index Terms-Wireless Sensing Sensor, IoT devices, Gesture Recognition, Occupancy Detection

\section{INTRODUCTION}

Human activity capturing and behavior modeling in residential environments draw increasing attention in communities because of its significant importance in healthcare [1], smart education [2] and human-machine interaction [3]. Occupancy detection and activity recognition are two essential tasks in these areas, particularly for their invaluable information in both long-term and short-time tasks. Compared with the traditional sensors like wearables [4], cameras [5], body sensor network [6], infrared imaging spectrometers [7], RF sensors are an emerging technology solution in the space. The reason is, in part, due to its unobtrusive characteristics and ubiquitous nature which can lead to the large coverage area and flexible deployments. Additionally, unlike camera systems, wireless signals are not able to generate images which alleviates many privacy concerns.

The fundamental concept of RF sensing is that when a person moves, the motion of his body will affect the com-

This work is part of the OPERA project funded by the UK Engineering and Physical Sciences Research Council (EPSRC), Grant No: EP/R018677/1

Wenda Li, Shelly Vishwakarma, Chong Tang, Kevin Chetty are with the Department of Security and Crime Science, University College London, London, WC1H 9EZ, UK (e-mail): wenda.li, s.vishwakarma, chong.tang.18, k.chetty@ucl.ac.uk.

Karl Woodbridge is with the Department of Electrical and Electronic Engineering, University College London, London, WC1H 9EZ, UK (email: k.woodbridge@ucl.ac.uk).

R. J. Piechocki is with Department of Electrical and Electronic Engineering, University of Bristol, UK (e-mail: r.j.piechocki@bristol.ac.uk). munication channel in terms of signal attenuation, frequency shift, and propagation paths. As a result, the time-varying communication channel is influenced by the physical movements and therefore can be used for monitoring purposes [8]. On the other hand, as a new technology, IoT network is designed to interconnect with various sensors, devices and applications [9], [10]. With the deep learning (DL) successful applications in the fields of image classification [7], RF transmissions in IoT networks show a great opportunity for passive sensing applications. However, there is still no mature solution to use these signals effectively.

One feasible approach is known as the Channel State Information (CSI) system. As a by-product of the WiFi network [8], CSI is used to estimate the quality of communication channels to facilitate reliable data transmissions [11]. The communication channel can be affected by the moving object which changes the CSI and can be potentially used for categorizing the object's activity [12], indoor localization [13] and occupancy detection [14]. CSI systems are designed to work for a specific WiFi standard, for example, the popular Intel $5300 \mathrm{WiFi}$ card [15] is designed for 802.11n only. However, IoT networks will be designed to use multiple communication standards such as Zigbee, Bluetooth, etc [16], to fulfill requirements in energy-saving, distance and throughput. The development cost of a CSI system that is compatible with all these different standards could therefore be prohibitive. In addition, CSI systems require high data throughput associated with active use of the wireless network in order to provide 
sufficient sensing performance [12], [17] which will not only interrupt the communication channel in IoT network but also drain more power from IoT devices [10].

On the other hand, Passive Radar (PR) systems also offer a number of advantages for sensing within an IoT network. PR systems do not transmit any signal but instead, use the third party signal source as an illuminator of opportunity. It has a long history in airborne detection and maritime surveillance [18] which exploits existing FM radio, television and DVB-T signals. These PR systems typically make use of relative narrowband signals for detecting large targets at long distances. Passive WiFi Radar (PWR) research has attracted lots of attention in recent years owing to the increased demand in near-field sensing applications. In work [19], authors demonstrate the feasibility of tracking drones, whilst work [20] counts the pedestrians in urban traffic scenarios. PWR systems have also been used in human presence detection [21] and activity recognition [22]. However, these PWR systems cannot be directly applied to IoT networks as the mechanisms of signal broadcast from IoT devices are rather different from that of a WiFi router. Also, variations in bandwidth, signal strength and data rate can significantly affect the sensing performance of PR systems.

The overview of purposed wireless sensing in IoT environment is demonstrated in the Figure in abstract. The physical configurations for PIoTR involve several pairs of WiFienabled IoT devices to be deployed in different places. These devices can be powered by Radio Frequency Energy Harvesting (RFEH) technique which deliveries energy wirelessly for sensors with a battery-less design. In such circumstances, PIoTR is designed with two modes to fully make use of RF signals from IoT devices. Firstly, coarse sensing mode uses the sparse WiFi signal for occupancy detection/roomlevel localization. To improve the sensitivity and increase the coverage area, multiple IoT devices are considered. In fine-grained sensing mode, the strong RFEH signal has been captured for high accuracy activity recognition not only in Line-of-Sight (LoS) but also in Through-The-Wall (TTW). PIoTR draws on classical PR techniques and adapts for IoT devices with different configurations depending on the signal strength, number of sources, etc.

Comparing to previous works [17], [22]-[24], the following contributions are made by this paper:

- Different from the above works on RF sensing, we use the uncontrolled transmission signals from commercial IoT devices as the source. This is more challenging than those specifically modified transmitters.

- Multiple IoT devices have been tested including Amazon Alexa, Google Nest Cam, and a RFEH transmitter. Based on the communication mechanism of these devices signal processing methods optimized for different frame rates were selected.

- The performance of PIoTR is supported by several experiments with acceptable results. PIoTR achieves $91 \%$ accuracy in room occupancy detection with sparse IoT signal and more than $99 \%$ with a continuous signal. The average accuracy in activity recognition also reaches $91.3 \%$.
The rest of this article is organized as follows. Section II introduces the IoT devices that used in this paper; Section III presents the signal processing and system implementation; Section IV presents the experimental results; discussions and conclusions are in Section V and VI.

\section{Related Works}

In this section, RF sensing techniques that have been applied to IoT devices are discussed, including the CSI system which works with WiFi systems, and PR systems which can work with multiple signal sources.

\section{A. Channel State Information System}

At the physical layer of wireless communications, CSI is useful information for environmental perception. It measures the changes in wireless signal due to the static and dynamic objects in terms of time, frequency and spatial domain. The different application requires specific signal processing and classification/estimation algorithms.

Device-free activity recognition is one of the applications by CSI systems. An early work [25] uses Short-Time Fourier Transform (STFT) to convert the CSI measurement into Doppler spectrogram. It works at a high frame rate at $2500 \mathrm{~Hz}$ and achieves $89 \%$ accuracy. However, this system only works on a pre-defined path and a pre-defined walking direction. Thus training is required for every new environment.

Another work [26] demonstrates a CSI system that contains three receiving channels at different angles and runs at a frame rate of $1,500 \mathrm{~Hz}$. The system claims a recognition accuracy of $96 \%$ and quantifies the relationship between CSI variation and human movement speed to eliminate the effect of the environment.

Fall detection is another important field for smart health. Work [27] develops a CSI system that is able to automatically detect falls from other activities. It calculates the power profile in time-frequency domain by exploiting the phase and amplitude changes in CSI measurements. This system works at a frame rate of $100 \mathrm{~Hz}$, however its performance is affected by the environment and user-antenna location.

CSI-based Breathing detection has been shown in [28], with a low frame rate of $20 \mathrm{~Hz}$. This system exams the user's location and body orientation based on the Fresnel model in an ellipse shape. However, its monitoring area is limited within the middle area between the transmitter and receiver.

More recently, a WiFi-enabled IoT system [29] was designed for occupant activity sensing. This system collects fine-grained CSI measurements and uploads them to a cloud server for processing. The system shows an accuracy of 96.8\% in occupancy detection and 90\% for six-class activity recognition. However, the system is built based on two WiFi routers with a frame rate of $500 \mathrm{~Hz}$ which can not represent the actual situation of IoT network.

As discussed above, CSI system is a by-product of WiFi network. Theoretically, it should work with the IoT devices that communicate via WiFi networks. However, it would be with a high cost to design a network card that is compatible 
with all the major WiFi standards, also those non-WiFi networks. In addition, CSI system normally requires a high frame rate for sufficient detection [25], [26], which are around 1000$2500 \mathrm{~Hz}$. This frame rate is very high and conflicts with some IoT devices which have a limited battery or energy-efficient design.

\section{B. Passive Radar System}

PR systems, with different mechanisms as CSI systems, have also been widely used for short-range RF sensing. The fundamental principle of PR system is to use the signal from a third-party transmitter and measure the time/phase difference between the signal arriving directly at the transmitter and the signal arriving via reflection from the object of interest. Different from CSI system, PR system does not have the acknowledgment about the preamble signal. It calculates the range (bistatic distance) and Doppler (bistatic velocity) information.

An early work utilizes WiFi transmissions with PR is described in [30]. It presents the feasibility detect a person at a stand-off distance $(12 \mathrm{~m})$ with a WiFi router under ThroughThe-Wall (TTW) scenario. However, the system needs a channel which directly attached to the WiFi router for perfect signal reconstruction.

Recently, PR system based on SDR platforms becomes more popular due to their flexibility in deployment and fast development. Work [31] presents a prototype base on the SDR platform with real-time ability by using a pipeline design for multi-core processing. The system has shown several preliminary results such as TTW activity recognition and finger gesture recognition. It is based on a classical passive radar configuration and assumes that the reference channel is stable, which may to ideal in real-world.

Our previous work [24] has demonstrated the possibility to use PR system for multiple detection purposes. The system can perform vital sign detection and activity recognition by adjusting the Doppler resolution. This is achieved by extracting the micro-Doppler as the representative for the chest motion and from torso and limbs. However, the system still requires a high frame rate for sufficient detection.

On the other hand, signals from Bluetooth Low Energy (BLE), in unlicensed 2.4Ghz frequency band, have also been studied for indoor localization. System [32] uses a fingerprinting technique to passive track the personnel's location depending on the variation in Received Signal Strength (RSS). $50 \mathrm{~Hz}$ of BLE beacon signal was set with a total of 19 BLE transmitters to cover an area of $750 \mathrm{~m}^{2}$. This system configuration is similar to an IoT network which includes multiple signal sources. However, the fingerprinting technique just like RSS data is highly sensitive to the surrounding environment and requires a considerable calibration process when the background is changed.

Note that, above works have made some changes to the $\mathrm{WiFi} / \mathrm{BLE}$ AP to enable a high frame/beacon rate. Also, works [24], [30], [31] attached an antenna to the WiFi router which assumes the transmitted signal can be perfectly reconstructed. However, such configurations are hard to apply in real-world
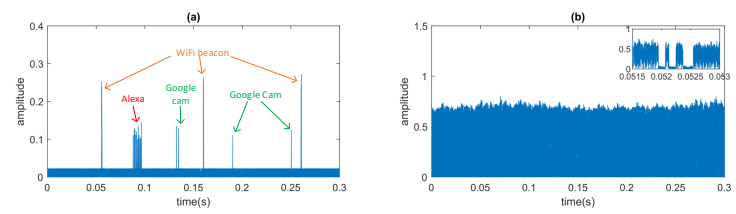

Fig. 1: An example of baseband signal from home IoT network (a) WiFi signal including Alexa and Google Cam and (b) RFEH signal, mini plot shows the zoom-in view

scenarios due to bandwidth is scarce in a communication network. Also, these systems are set up with active radar-like geometry that transmitter and receiver are on the same side, are too optimal to be real.

\section{Preliminary Investigations Of Iot Devices}

In this section, we investigate the communication mechanism of three popular IoT devices and explore their potential in RF sensing. Different from previous works, we select three commercial IoT products as the illumination sources without any specific modification. These devices have been widely used in residential and domestic applications for communication and wireless energy. Multiple WiFi connected devices can increase the frame rate to improve detection performance, however, their effective signals depend on the amount of internet usage. In comparison, a RFEH transmitter, aims reliable energy delivery [33], has also been used which outputs a powerful and constant signal as the comparison for IoT-based devices. The communication mechanisms of these devices are discussed as below:

- WiFi router provides a gateway to the internet for other devices. WiFi router constantly broadcasts beacon signals to announce its presence which is normally fixed at $10 \mathrm{~Hz}$ [22]. It can achieve high frame rates when there are is internet usage [12], [34]. The maximum transmission power of a router is $100 \mathrm{~mW}$.

- Amazon Echo is a cloud-based voice service that provides human-machine interaction through its virtual assistant Alexa i.e answering questions and playing music. It is observed that during idle status, the Echo is in constant contact with WiFi router at a very low frame rate frequency of around $0.1 \mathrm{~Hz}$. This rate increases when the device is active (for example playing music or radio), which reaches roughly $1-2 \mathrm{~Hz}$.

- Google Nest Cam provides remote monitoring, it uploads video data to a cloud server to be viewed by the user. Our initial observations indicate that it has a constant upload rate at around $10 \mathrm{~Hz}$, providing a constant WiFi signal to be used for RF sensing.

- RFEH transmitter [35] delivers wireless energy to surrounding IoT devices that are not equipped with a battery. It operates at the $915 \mathrm{MHz}$ ISM band, with a maximum output power of $1 \mathrm{~W}$. Therefore, this powerful and consistent signal can be used for fine-grained sensing by PIoTR. Its protocol for communications is to employ Direct Sequence Spread Spectrum (DSSS) modulation. 


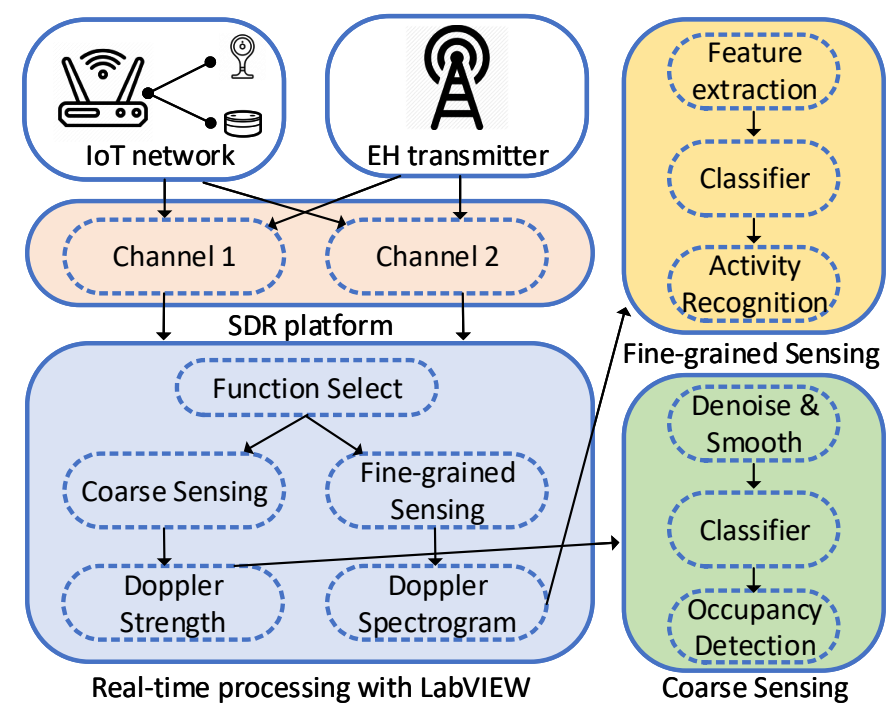

Fig. 2: Overview of the system

An example of baseband signal from a home IoT network is shown in Fig 1(a) WiFi signal and (b) RFEH signal. As it can be seen, the frame rate is very low even there are two WiFi devices are operating at same frequency band. As a result, the sparse WiFi signal is not sufficient for fine-grained sensing tasks like activity recognition. Additionally, each device has different signal strength due to its location and output power. On the other hand, RF signal from a RFEH transmitter is with significantly higher power and is more consistent than the WiFi network. There are only small-time gaps among packages (as shown in the mini figure), and with very small fluctuation in signal strength. This provides a unique solution for RF sensing.

\section{System Design \& Signal Processing}

\section{A. System Overview}

The overall block diagram of PIoTR is presented in Fig 2. It contains mainly three parts: RF front-end which samples the wireless signal, signal processing which generates Doppler information and classifiers which are implemented for different tasks.

An important task is that the system should be able to handle different frequency bands, such as the $2.4 \mathrm{GHz}$ in WiFi and $915 \mathrm{MHz}$ in EH. This capability sets it apart from CSI-based systems [29], [34] which only applies to WiFi standards.

For this reason, we built the system on a Software Defined Radio (SDR) platform which can adapt its operational frequency as required. The front-end hardware contains two NI USRP-2921 [36], each equipped with a tunable RF receiver to acquire the wireless signal. Two directional antennas are used. The collected raw data is then transferred to a computing unit (a laptop in this work) through Ethernet.

To make full use of the available signal sources, PIoTR system has two sensing modes: sparse sensing which uses lowduty cycle WiFi signals from Amazon Alexa and the Google Nest Cam for coarse occupancy detection; and fine-grained sensing which uses strong RFEH signals for a more challenging task like activity recognition. In coarse sensing, tasks like occupancy detection and room-level localization, Doppler strength is calculated as representative of the human presence. In fine-grained sensing, tasks like activity recognition and event classification, require advanced signal processing and classifiers to achieve sufficient performance. The signal processing for generating the Doppler spectrogram has been designed with a low-complexity design [22], which can be processed in real-time within LabVIEW. Classifiers due to their high computational complexity are implemented off-line processing. PIoTR first select sensing mode depending on the effective signal, then perform signal processing accordingly.

One of the challenges is to handle the wireless signal with unknown modulation methods, such as OFDM in WiFi signal and DSSS in RFEH signal. It would be time-consumed to implement a specific filter for each signal. Therefore, we employ the generic cross-ambiguity function [34] which correlates the wireless signal in time domain rather than calculating channel impulse response with pilot signal in frequency domain, as implemented in CSI-based sensing systems [8]. This method has been broadly applied to multiple types of signal sources in passive radar [18], [19]. Such an approach would enable the PIoTR system to compatible with different types of signal sources or standards without demodulation, consequently, reduces the system complexity and hardware cost.

\section{B. Coarse Sensing: Room Occupancy Detection}

Occupancy detection provides invaluable information for smart health, as it generates knowledge about the resident's life patterns, especially those who need long-term monitoring or care. Classical CSI-based methods [25], [29], [34] require high frame rates $(1500 \mathrm{~Hz}-2500 \mathrm{~Hz})$ which occupy significant bandwidth for an IoT network. In comparison, our PIoTR system is designed to work with low frame rates for both IoT devices and WiFi APs (as shown in Fig 1(a)). Occupancy detection is presented as an example of coarse sensing.

In its operation, a PR system requires a minimum of two coherent receivers; one termed the (reference) channel, and the other termed the (surveillance) channel. The reference antenna is directed towards the transmitter (signal source) to obtain a copy of the original signal, whilst the surveillance antenna is towards the areas of interest. However, due to the multiple signal sources in IoT network, it is not a feasible solution to recreate the transmitted signal from each individual device. Thus, we replace the concept of surveillance and reference channel, with the idea to compare range and Doppler variation between two channels. In this work, Cross Ambiguity Function (CAF) has been used to correlate the signal from two channels in order to generate range-Doppler plots. Let the wireless signal received at two channels as $Y_{1}(t)$ and $Y_{2}(t)$, the CAF surface can be calculated as:

$$
\begin{aligned}
C A F\left(\tau, f_{d}\right)=\sum_{i=0}^{M-1} \int_{0}^{T_{i}} Y_{1}\left(t-\tau_{1, i}\right) e^{j 2 \pi f_{d_{1, i}} t} \\
Y_{2}^{*}\left(t-\tau_{2, i}\right) e^{j 2 \pi f_{d_{2, i}} t} d t
\end{aligned}
$$



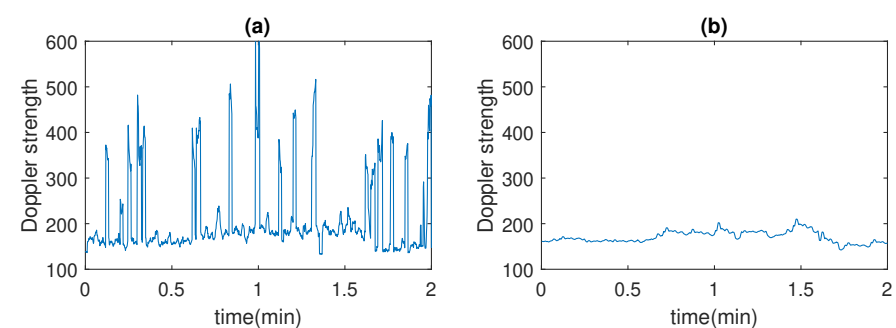

Fig. 3: Comparison of (a) raw and (b) denoised Doppler strength
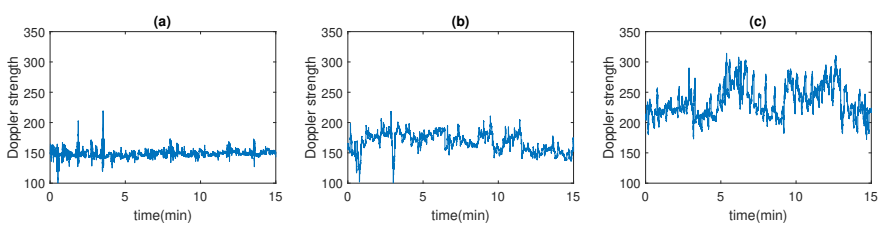

Fig. 4: Doppler strength for (a) an empty room, (b) one person resting on a bed (reading on tablet) and (c) one person in living room (normal activity)

where, $\tau_{1, i}$ and $\tau_{2, i}$ represents the time delay received from $i$ th IoT device at channel 1 and $2, f_{d_{1, i}}$ and $f_{d_{2, i}}$ represent the Doppler shift received from $i$-th IoT device for total $M$ devices. $T_{i}$ is the integration time, and $*$ denotes the complex conjugate. This equation calculates the range/Doppler difference among channels. It cannot accurately calculate the range/Doppler profile from moving targets due to having no reference signal available. However, this output can still though be used to infer the occupancy of a room. To enable real-time processing to generate Doppler spectrograms, a pipeline structure and batch processing have been implemented. Details of this structure is followed by the suggestion from [24].

Afterwards, Doppler profiles from each individual CAF surface are collected and combined to generate a time-varied Doppler spectrogram. One limitation of this approach is that only coarse Doppler information can be extracted, which will result in high levels of noise and sidelobes. We attribute this effect to both poor correlations with multiple sparse WiFi signals and interference from imbalanced signal strength.

Thus, we further extract the Doppler strength $D_{s}$ from spectrogram $D$ as the indicator for occupancy. Doppler strength is calculated as:

$$
D_{s}(t)=\sum_{f_{d}=-N_{b} / 2}^{N_{b} / 2} D\left(f_{d}, t\right)
$$

where $N_{b}$ is the number of Doppler bins which defines the maximum detectable velocity. Once the Doppler strength has been calculated, it is leveraged for classification. However, it is observed that this data has intrinsic noise and filters in time domain are required for good classification performance.

1) Outlier Removal: The correlation in Eq 1 has high levels of noise due to the sparse WiFi signal (as shown in Fig 1(a)). A number of outliers span the entire dataset with considerably high amplitudes that need to be detected and removed. We utilize a threshold approach estimated by Kmeans algorithm to guarantee the quality of Doppler strength at each time index and maintain a fixed interval between successive measurements.

2) Smoothing: Our initial measurements have revealed that the transmission power from IoT devices is time-varying. To deal with it, we use a Savitzky-Golay (SG) filter to smooth the data. The cleaned Doppler strength $\hat{D}_{s}$ is calculated as:

$$
\hat{D}_{s}=\sum_{i=-1 / 2 M_{s}}^{1 / 2 M_{s}} C(i) D_{s}(t)
$$

where $C(i)$ is the coefficients and $M_{s}$ denotes the SG window length which was fixed at $1 \mathrm{~s}$ in this work. The output value is smoothed at the central point of $i=0$. The comparison of raw and denoised Doppler strength data is shown in Fig 3.

Afterwards, we present measurements in three different scenarios: (a) an empty room, (b) one person resting on a bed, and (c) one person spending time in a living room as shown in Fig 4. For the empty room, the Doppler strength is observed to be very stable and with a low value due to no Doppler (activity) being detected in the room. The Doppler strength becomes much stronger in (c) with significant variation when the subject was carrying out everyday activities in the living room like cooking and cleaning. In comparison, Doppler strength in (b) is weaker with much less variation when the subject remained relatively stationary whilst reading in a bedroom. There are still considerable variations when compared to the empty room in (a). Based on these observed differences we hypothesise that occupancy detection is achievable using PIoTR by exploiting the variations in Doppler power.

The extracted Doppler strength is considered as time-series data. Based on this fact, we used the Long Short-Term Memory (LSTM) network [37] as the classifier. The structure of LSTM net allows it can learn the temporal dynamic behavior that has been applied in many radar applications with great success [38]. In this work, we built a two-layer bidirectional stacked LSTM net, which is designed to effectively learn the data temporal correlation forwards and backwards. Afterwards the data is reshaped into a form of $\left(N, L, H_{i n}\right)$, where $N$ refers to the number of data, $L$ means the length of data and $H_{i n}$ is the number of input features at each time step. Furthermore, we set the hidden size, $H_{\text {out }} \mathrm{m}$ of LSTM to 32 , which means features at each time step will be projected to the 32 dimensions. Therefore, the output from the net has a shape of $\left(N, L, 2 * H_{\text {out }}\right)$. Herein, due to the bidirectional feature, the outputs at each time step is the concatenation of forwards and backwards learning results so that there is the doubled hidden size above. For the classification task, we only used the output from the final time step which has a shape of $\left(N, 1,2 * H_{\text {out }}\right)$. Finally, these extracted features are fed into a three-layer fullyconnected network to predict the class of each data. During the training phase, we used the SGD optimizer with 0.001 learning rate and 0.9 momentum. The objective function used is Cross Entropy loss. Moreover, we set the batch size to be 32 . 


\section{Fine-Grained Sensing: Activity Recognition}

Human activity recognition is an important topic in humanmachine interface for a wide range of applications such as remote detection and behavior analysis. As discussed above, human activity can be captured by wireless signals and characterized by time-domain features. The powerful RFEH signal is naturally compatible with this topic.

Different from WiFi network, RFEH transmitter is considered a single source in fine-grained sensing. Therefore, the transmitted signal is needed as a reference to extract relative time delay and Doppler shift from the reflected signal. For this reason, the concept of surveillance and reference channel is adopted for fine-grained sensing, where surveillance channel collects the reflected signal from personnel and reference channel records the original transmitted RFEH signal. Let the signal from surveillance channel as $S_{\text {sur }}(t)$ and from reference channel as $S_{r e f}(t)$, the CAF can be calculated as:

$$
C A F\left(\tau, f_{d}\right)=\int_{0}^{T_{i}} S_{\text {sur }}(t) S_{r e f}^{*}(t-\tau) e^{j 2 \pi f_{d} t} d t
$$

where $C A F\left(\tau, f_{d}\right)$ is a $2 \mathrm{D}$ surface contains range $\tau$ and Doppler $f_{d}$ information.

The surveillance channel receives reflected signal which contains Doppler shifted target echoes as well as contributions from surrounding clutter. Thermal noise in the receiver is also present. The expression for surveillance channel can be expressed as:

$$
S_{\text {sur }}(t)=S_{d s i}(t)+S_{\text {clutter }}(t)+S_{\text {tar }}(t)+n(t)
$$

where $S_{d s i}$ represents the direct signal interference, $S_{\text {clutter }}$ represents the reflected signal from static clutter, $S_{t a r}$ represents the signal from dynamic objects and thermal noise is $n(t)$. Eq 4 may break down if the power from $S_{d s i}$ is much stronger than the $S_{t a r}$. This is a common case in real environment where the direct signal is stronger than reflected signal. As a result, the desired Doppler pulse from dynamic objects may be buried by the Doppler pulse due to direct signal. For this reason, it is necessary to remove this unwanted direct signal from the CAF surface.

In this work, the CLEAN algorithm introduced by [20] has been adopted. It uses a similar approach to the CAF processing but only correlates the signal from reference channel. This generates a self-CAF surface that contains the Doppler pulse created by the direct signal. By suppressing the self-CAF surface from $\mathrm{Eq} \mathrm{4}$, the buried Doppler pulse is revealed. Let $C A F^{k}\left(\hat{\tau}, \hat{f}_{d}\right)$ represents the cleaned CAF surface at $k_{t h}$ iteration, it can be written as:

$$
C A F^{k}\left(\hat{\tau}, \hat{f}_{d}\right)=C A F^{k-1}\left(\tau, f_{d}\right)-\alpha^{k} C A F_{\text {self }}\left(\tau-T_{k}, f_{d}\right)
$$

where $\alpha^{k}$ is the maximum absolute value of $C A F^{k}\left(\tau, f_{d}\right)$, $T_{k}$ is the phase shift factor and refers to $\alpha^{k}$. The phase of $C A F^{k}\left(\hat{\tau}, \hat{f}_{d}\right)$ is shifted through a multiplication with a complex phasor $e^{j \Delta \phi}$, where $\Delta \phi$ is the phase difference between $C A F^{k}\left(\hat{\tau}, \hat{f}_{d}\right)$ and Doppler pulse along zero bins.
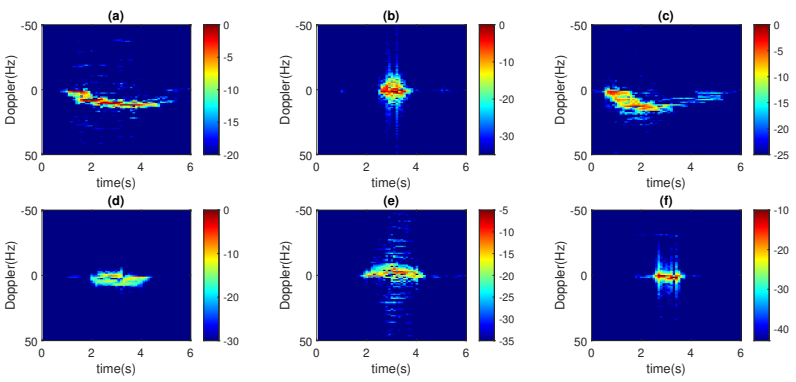

Fig. 5: Doppler spectrograms for six human activities, (a) walk away, (b) jump, (c) run, (d) sit, (e) stand and (f) turn

In the final step, like coarse sensing, a noise reduction is required to remove the unexpected pulses due to incorrect correlation which occurs periodically and to improve the pulseto-background ratio. The Constant False Alarm Rate (CFAR) method has been adopted which calculates a threshold mapping based on the moving average of the cleaned CAF surface. As suggested by [39], this threshold is obtained by estimating the power of noise during a period of static background. Doppler pulses that are lower than the threshold mapping are considered as noise, otherwise they are considered as being part of an 'activity' and are be included in the Doppler spectrogram.

Fig 5 shows Doppler spectrograms captured from six activities, including walking, jumping, running, sitting, standing, and body turning. As can be seen, each activity results in differing micro Doppler signatures which manifest in both the positive and negative Doppler regions as well as temporally. These variations provide rich information corresponding to the velocity and direction of the dynamic objects and therefore can be used for fine-grained activity recognition. For example, running has more Doppler shift components than walking which is attributed to faster periodic torso speeds. Jumping has a Doppler signature in both the positive and negative domain owing to the upward and downward motion of the body. Sitting and standing have opposite Doppler signatures for different body directions, while body turning has a distinctive shape as part of its signature.

Afterwards, Doppler spectrograms were passed to a VGG16 network [40] to be further classified. The size of input data (a Doppler spectrogram) is $(100,40,1)$. Therefore, to make our input data fit the network, we modified the original input channel number of the first convolutional layer from 3 to be 1 . Then the number of neurons in the fully-connected layers of VGG16 are also correspondingly changed with an epoch time of 300. During the training phase, we used SGD optimizer with 0.0005 learning rate and 0.9 momentum. The batch size is set to be 32 and the loss function we used is Cross Entropy loss.

\section{Interference from Frame Rate}

Frame rate is an important factor that affects the sensitivity of PIoTR. It defines the mode to choose between coarse sensing or fine-grained sensing. Here we measure the effect of frame rate on the PNR between the dominant Doppler peak 


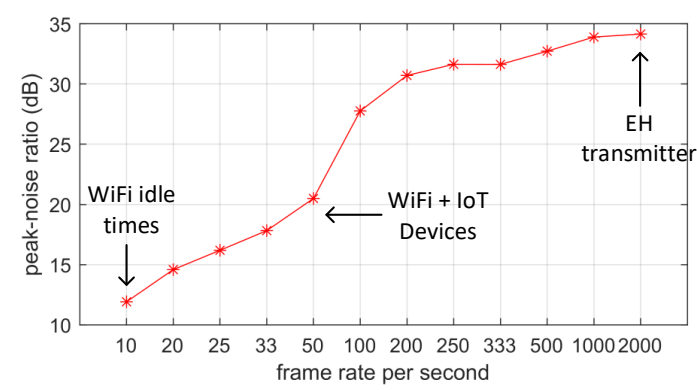

Fig. 6: Effect of frame rate on Peak-Noise Ratio (PNR)

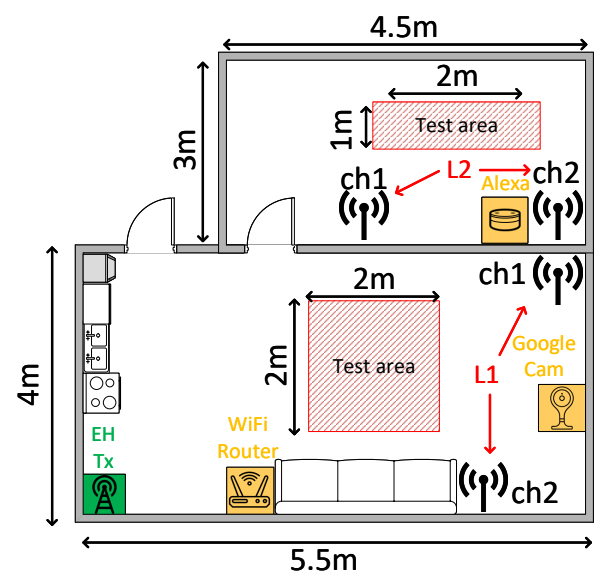

Fig. 7: Experiment layout in a one-bedroom flat, ch1 and ch2 represent channel 1 and 2, and L1 and L2 represent layout 1 and layout 2

and rest of the spectrogram. This Doppler peak is calculated using the self-ambiguity function, which correlates with the signal itself. Higher PNR means better quality Doppler spectrograms. The PNR versus frame rate is shown in Fig 6. The baseline is when WiFi router in idle mode where only beacon signals are broadcast at a frequency of $10 \mathrm{~Hz}$. Adding other IoT devices, such as one Amazon Alexa and one Google Cam, can increase the frame rate to $50 \mathrm{~Hz}$. However, the PNR is still not enough for fine-grained sensing at this frame rate. Therefore, coarse sensing is enabled within the WiFi network. PNR becomes much sufficient and stable after $200 \mathrm{~Hz}$ of frame rate. A small improvement can be seen at $2000 \mathrm{~Hz}$ frame rate from RFEH transmitter. The trade-off between PNR and frame rate is therefore estimated at around $200 \mathrm{~Hz}$ for the optimized detection performance. Frame rate below $200 \mathrm{~Hz}$ will be used for coarse sensing, and above $200 \mathrm{~Hz}$ could be used for finegrained sensing. Note that, parameters like data rate, frame size and bandwidth can also affect the frame rate in WiFi network.

\section{EXPERIMENTAL RESULTS}

In this section, experimental results are presented to show the performance of PIoTR for both occupancy detection and activity recognition.
TABLE I: System parameters associated with the coarse and fine-grained sensing modes

\begin{tabular}{|l|l|l|}
\hline & Coarse sensing & Fine-grained sensing \\
\hline Signal source & $\begin{array}{l}\text { WiFi router/Amazon } \\
\text { Alexa/Google Cam }\end{array}$ & RFEH transmitter \\
\hline Number of sources & $\begin{array}{l}\text { multiple (three de- } \\
\text { vices in this work) }\end{array}$ & single \\
\hline Frame rate & $25-30 \mathrm{~Hz}$ & $>2000 \mathrm{~Hz}$ \\
\hline Output power & $\begin{array}{l}<100 \mathrm{~mW} \text { (varied de- } \\
\text { pending on device) }\end{array}$ & $<1 \mathrm{~W}$ \\
\hline Carrier frequency & $2.4 \mathrm{GHz}$ & $915 \mathrm{MHz}$ \\
\hline Bandwidth & $20 \mathrm{MHz}$ & $20 \mathrm{MHz}$ \\
\hline Iteration time $\left(T_{i}\right)$ & 1 second & 1 second \\
\hline $\begin{array}{l}\text { Number of Doppler } \\
\text { bins }\left(N_{b}\right)\end{array}$ & 40 & 100 \\
\hline Application & occupancy detection & activity recognition \\
\hline Antenna & directional (14dB) & directional (8dB) \\
\hline
\end{tabular}

\section{A. Experiment Setup}

The experiment layout is shown in Fig 7 in a one-bedroom flat, consists of a living room and a bedroom. To test the PIoTR in both LoS and TTW conditions, two system layouts were tested. Channels in layout 1 were located in the two corners of the living room and channels in layout 2 were located in two corners of the bedroom. An Amazon Alexa was located in bedroom and playing music whilst a Google Nest cam was located in living room and configured to recording video. The WiFi router was located on the opposite side of the living room near the sofa. These three devices combined can be considered as a simple IoT network. Moreover, a RFEH transmitter was located in the bottom-left corner of the living room. This is to cover the entire flat for RFEH transmitter delivers wireless energy to IoT sensors. The frame rate in coarse sensing is about $25-30 \mathrm{~Hz}$, whereas in finegrained sensing is about $3 \mathrm{kHz}$. Note that, all devices used in this work were in normal operation order which did not include any specific configuration in firmware or hardware. For clarify, Table I summarizes the system parameters for PIoTR.

Total four volunteers (three male and one female) were involved, including six classes in occupancy detection and six classes in activity recognition.

\section{B. Coarse Sensing: Occupancy Detection}

Dataset for coarse sensing includes 360 minutes continuous measurement as 6 classes x 30 minutes each measurement x 2 layouts. Volunteers were asked to keep active to best stimulate as dynamic objects. This includes 'rapid' activities like walking, as well as slow activities like reading and working in front of a computer.

For all measurements, we split the long Doppler strength data into short samples with a duration of 30 seconds. This gives total 720 samples in coarse sensing. The focus is to decide whether there are people occupancy within 30 seconds. All classifications follow: $80 \%$ samples for training and $20 \%$ samples for testing. A five-fold cross validation was applied. Three metrics were calculated to evaluate the accuracy of occupancy detection as follows:

- Overall Accuracy $(\mathrm{OA})=$ correct detected samples $/$ total samples 


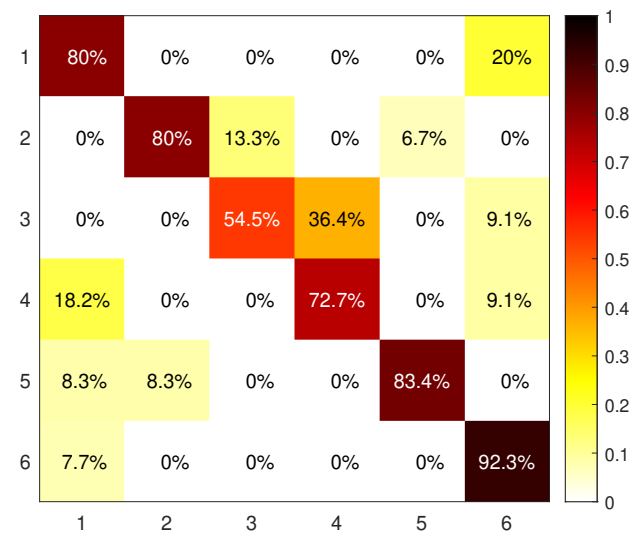

Fig. 8: Confusion matrix of room-level localization: Class 1 unoccupied home; Class 2 one person in the bedroom, one person in the living room; Class 3 multiple people in the living room; Class 4 multiple people in the bedroom; Class 5 one person in the bedroom; Class 6 one person in the living room.
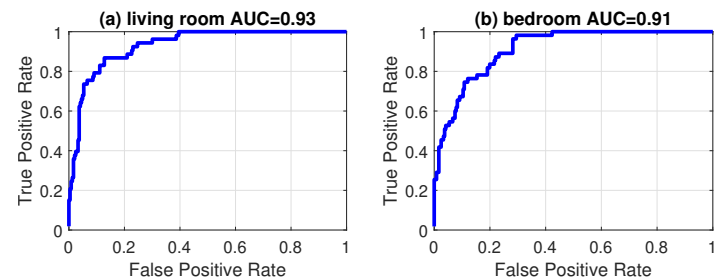

Fig. 9: Receiver Operating Characteristic (ROC) curve for occupancy detection (a) living room and (b) bedroom.

- Occupied Detection Accuracy $(\mathrm{ODA})=$ correct detected occupied samples / true total occupied samples

- Unoccupied Detection Accuracy $(U D A)=$ correct detected unoccupied samples / true total unoccupied samples

1) Room-level Localization: In the following test, we exam the feasibility of PIoTR for room-level localization. In this test, we mixed the samples from two layouts. The confusion matrix for six classes is shown in Fig 8. The best performance cases are class 5 and 6; both are the single person class and reach an accuracy of $83.3 \%$ and $92.3 \%$. These results suggest that PIoTR can deliver high accuracy in the detection of individual person. In comparison, the worst performance occurs for class 3 at only $54.5 \%$ with many samples misclassified into class 4 . This is due to the activity model from multiple people being more complex than that for a single person. We also provide ROC curves of occupancy detection from two rooms, which are shown in Fig 9. Overall, gain in true positive rate is greater than $90 \%$, while Area Under Curve (AUC) of living room is slightly better than bedroom. This is because the WiFi router was located in living room and gave better opportunistic for illumination.

2) Single \& Multiple People Occupancy Detection: In this test, we compare the performance of occupancy detection for single and multiple people by two layouts. Fig 10 shows the measurement metrics. Single person has OA at around $88 \%$, whereas multiple people have OA at around 90\%. ODA and UDA of multiple people are also better than that in single

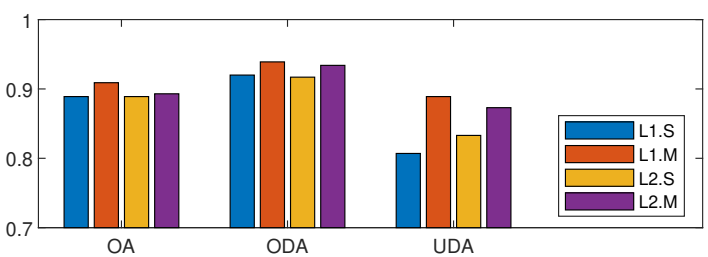

Fig. 10: Performance of occupancy detection for single (S) and multiple (M) people

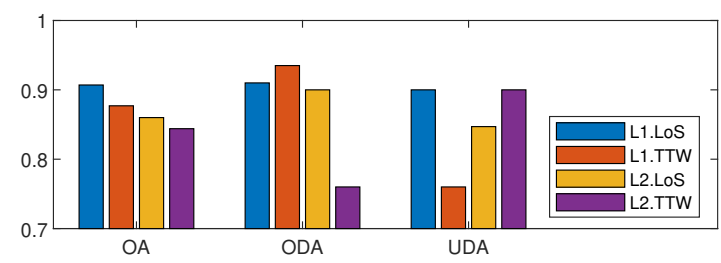

Fig. 11: Performance of occupancy detection in living room and bedroom

person. These results demonstrate that occupancy detection for multiple people is slightly better than that for single person. The reason is due to multiple people have a larger combined radar cross section which is more likely to be detected. It is also observed that all ODAs are better than UDA. This indicates PIoTR trend to make false alarms in unoccupied room. Visually inspecting the data, these noise in Doppler may introduced by imperfect correlation in $\mathrm{CAF}$ and power imbalance.

3) LoS \& TTW Occupancy Detection: In this test, performance for LoS and TTW scenarios are calculated separately by two layouts. The measurement metrics are shown in Fig 11. The overall detection performance in $\operatorname{LoS}$ is generally better than that in TTW as expected. Also, layout 1 has slightly better accuracy than layout 2 . We attribute this to the location of the WiFi router (in living room). Since WiFi router is a more stable signal source when compared to IoT devices that largely depend on internet usage. In contrast, only one device was in bedroom which lower PIoTR's accuracy.

\section{Fine-Grained Sensing: Activity Recognition}

Fine-grained sensing was verified with 6 different activities as indicated in Fig 5. PIoTR was tested following the configuration in Fig 7, where the RFEH transmitter was located at the left-bottom corner. A total of 480 samples were collected from four people, each with a window length of 4 seconds. We randomly select $80 \%$ of the dataset for training and used the remaining 20\% for testing. Three metrics, precision (positive predictive value), recall (sensitivity) and F1 (weighted average of precision and recall) were calculated to assess the accuracy of occupancy detection as follows:

$$
\begin{aligned}
& \text { - } \text { precision }=\mathrm{TP} /(\mathrm{TP}+\mathrm{FP}) \\
& \text { - recall }=\mathrm{TP} /(\mathrm{TP}+\mathrm{FN}) \\
& \text { - } \mathrm{F} 1=2 \times(\text { precision } \times \text { recall }) /(\text { precision }+ \text { recall })
\end{aligned}
$$

where, TP, FP, and FN represent true positive, false positive, and false negative, respectively. 


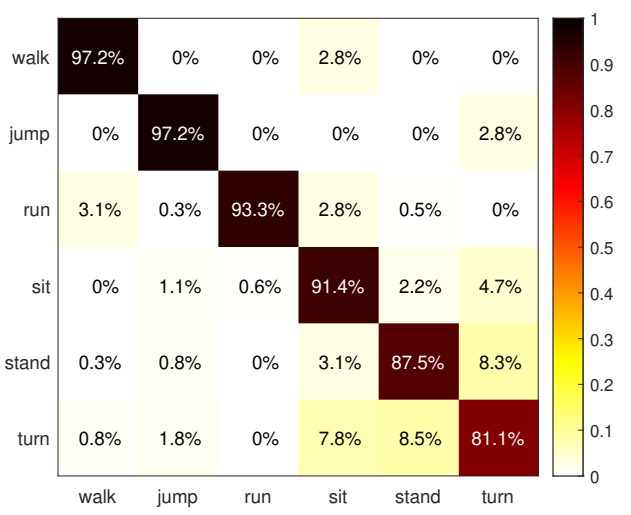

Fig. 12: Confusion matrix of activity recognition
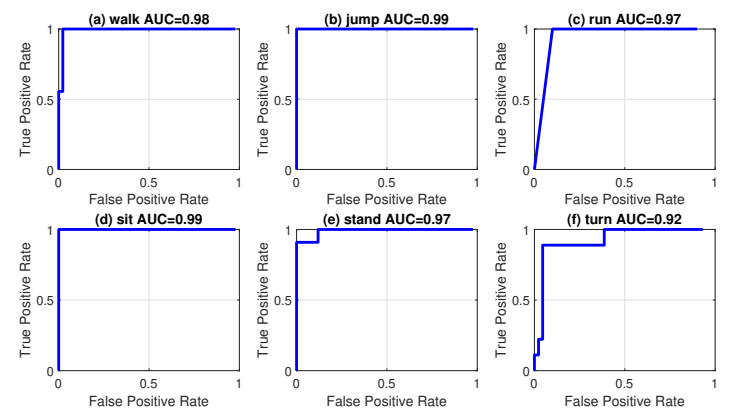

Fig. 13: ROC curves for each activities.

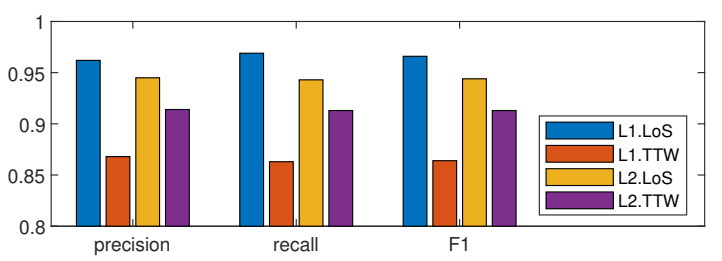

Fig. 14: Precision, Recall, and F1 results for activity recognition in living room and bedroom

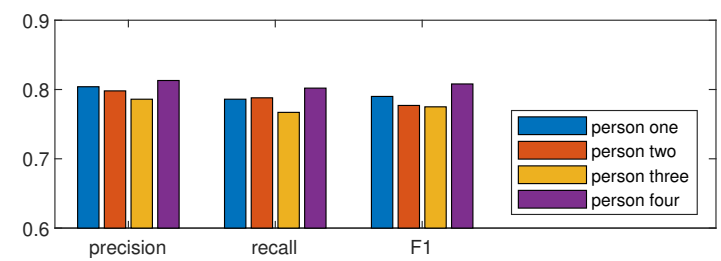

Fig. 15: Precision, Recall, and F1 results for inter-person recognition

TABLE II: Performance Comparison in different Locations

\begin{tabular}{|l|l|l|l|l|}
\hline $\begin{array}{l}\text { Testing lo- } \\
\text { cation }\end{array}$ & $\begin{array}{l}\text { LoS in liv- } \\
\text { ing room }\end{array}$ & $\begin{array}{l}\text { TTW in } \\
\text { bedroom }\end{array}$ & $\begin{array}{l}\text { LoS in a } \\
\text { lab }\end{array}$ & $\begin{array}{l}\text { TTW in an } \\
\text { individual } \\
\text { room }\end{array}$ \\
\hline $\begin{array}{l}\text { Distance to } \\
\text { transmitter }\end{array}$ & $5 \mathrm{~m}$ & $8 \mathrm{~m}$ & $6 \mathrm{~m}$ & $6 \mathrm{~m}$ \\
\hline Dataset size & 240 & 240 & 180 & 220 \\
\hline Precision & $96.2 \%$ & $86.8 \%$ & $94.7 \%$ & $84.3 \%$ \\
\hline Recall & $96.9 \%$ & $86.3 \%$ & $93.5 \%$ & $82.1 \%$ \\
\hline F1 & $96.6 \%$ & $86.4 \%$ & $94.1 \%$ & $83.2 \%$ \\
\hline
\end{tabular}

1) Overall Recognition Accuracy: Fig 12 presents the confusion matrix of activity recognition showing an overall accuracy of $91.3 \%$. As the figure shows, the best performing activities are walking and jumping, which both reach 97\% accuracy. This is due to their relative unique Doppler signatures that are easier to distinguish by the classifier. The running, sitting and standing activities all achieved an accuracy around 90\%. The worst performing activity was the body turning, which gave just over $80 \%$ accuracy. There are several misclassifications amongst the sitting, standing, and turning activities. The reason is believed due to their similar activity duration and weak Doppler signature. For example, walking and running both have higher velocity than the rest which makes them very easy to distinguish. In comparison, sitting, standing and turning have much weaker and shorter Doppler signatures as shown in Fig 6. ROC curves for each activity are shown in Fig 13. Due to higher accuracy, most AUC values in activity recognition are above 0.97 with exception of turn which is 0.92 . The results are consistent with our analysis on Fig 12.

2) Recognition Accuracy in LoS and TTW: In this test, samples from the LoS and TTW are trained and tested separately. The overall values of the precision, recall and F1 results are shown in Fig 15. As expected, LoS has better accuracy than TTW in both layouts, with the highest values in precision, recall and F1. Benefited from the high transmit power from the RFEH device, there is only a slightly downgraded performance under TTW condition. TTW in layout 1 has the worst performance, this is because the RFEH signal penetrated the wall twice (outward and reflection), which had been significantly attenuated.
3) Recognition Accuracy of Inter-Person: Here, we evaluate recognition accuracy between people by testing samples from one volunteer and training samples from the other two volunteers. This test is more realistic as in many scenarios, the classifier does not have a sample from unknown subjects and has to rely on the pre-trained classifier. The classification results are shown in Fig 15. As the figure shows, classification performance is similar for all four people at around $80 \%$. This downgrade in accuracy is due to the differences in Doppler signature between training and testing volunteers. Nevertheless, the system can still deliver good accuracy to recognize activities from a new user base on the previous training from known samples.

4) Recognition Accuracy in Different Locations: Finally, we further verified the performance of PIoTR in different locations including: a LoS scenario in a lab $(7 \mathrm{~m} \times 6 \mathrm{~m})$ and a TTW scenario in an individual room $(4.3 \mathrm{~m} \times 4.6 \mathrm{~m})$. The same activities were carried out in both experiments and a RFEH transmitter was used as the signal source. The classification process for each dataset was same as described above. Table II presents the details of two experiments and their classification results, together with that information from Fig 14. In general, LoS gives better performance than the TTW as the result can achieve more than 92\%, while in TTW can achieve around $83 \%$. Additionally, these results demonstrate PIoTR can deliver stable performance in different scenarios. of a stronger direct signal. Classification accuracy in LoS 

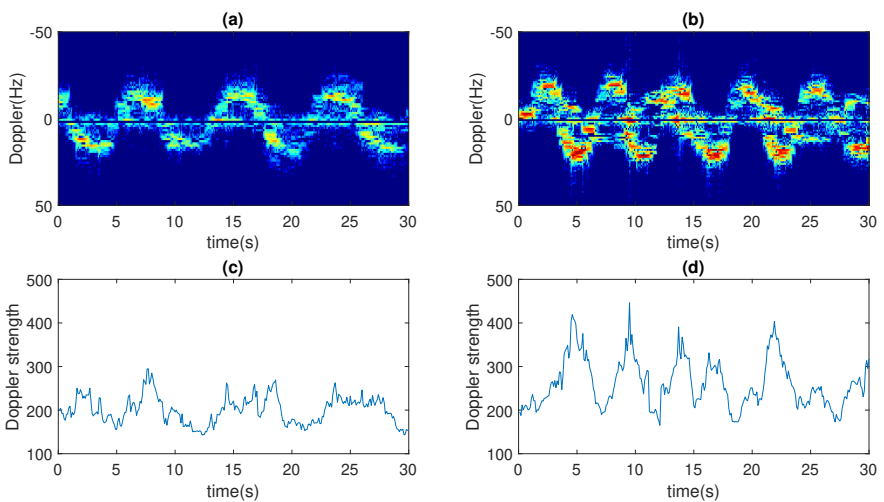

Fig. 16: Doppler spectrogram of (a) single person walking, (b) two people walking, and corresponding Doppler strength in (c) and (d), respectively.

TABLE III: Occupancy detection by fine-grained sensing

\begin{tabular}{|l|l|l|l|}
\hline & flat & living room & bedroom \\
\hline OA & $99.6 \%$ & $99.7 \%$ & $99.5 \%$ \\
\hline ODA & $99.7 \%$ & $100.0 \%$ & $99.4 \%$ \\
\hline UDA & $99.5 \%$ & $99.4 \%$ & $99.6 \%$ \\
\hline
\end{tabular}

\section{Fine-Grained Sensing for Occupancy Detection}

On the other hand, fine-grained sensing can be easily applied for occupancy detection. Fig 16 presents the Doppler spectrogram captured by walking from (a) single person and (b) two people walking, and corresponding Doppler strength. There are obvious differences in Doppler signatures between the two spectrograms. A single person shows a simple Doppler signature with a single trajectory, whereas two people have more a complicated Doppler signature as a combination of two trajectories. Afterwards, same strategy has been applied for occupancy detection.

Dataset in Section IV-C jointly with an empty room measurement had been used to test fine-grained sensing in occupancy detection, the performance is shown in Table III. Benefit by the powerful RFEH signal, the detection rate of OA, ODA and UDA are exceptional, where all of them are above $99 \%$. Thanks to high frame rate in RFEH transmitter, fine-grained sensing generates much better Doppler features than coarse sensing and therefore with better performance.

\section{Discussions}

\section{A. System Comparison}

Compare with the state-of-the-art WiFi CSI systems like TW-See [12], Wideep [13] and CsiGAN [34] which are biproducts of communication technique, PIoTR has different mechanisms. Firstly, PIoTR is designed with passive radar technique which is compatible with different types of signal sources [18], not limited to WiFi signal. Consequently, PIoTR is more suitable for IoT network which is embedded with various communication protocols [16]. Additionally, PIoTR generates Doppler information which does not affect by the background scatter. This means no background calibration is required which simplifies the system design. Moreover, CSI systems [12], [34] generally need to trigger high frame rate for sufficient detection which is not practical in real applications. In comparison, PIoTR does not interference the air travel in the communication channel since it passively uses the RF signal and has no direct control on the signal source. This is more suitable to the IoT network in terms of energy efficiency, bandwidth usage and system design [10]. However, PIoTR's 'passive' approach also means its performance is not guaranteed but depends on the frame rate (effective WiFi signal) in IoT network. A stable signal source, like RFEH transmitter, is therefore essential for PIoTR.

\section{B. Stationary Person Detection}

The key principle of PIoTR is to detect Doppler shifts resulting from moving objects, however this may not apply to stationary person who may be resting, reading, watching television, etc. Consequently, PIoTR may not perform well when the object is in stationary or with very low velocity. Additionally, without background scanning, extraction of stationary person from the static background has not been fully explored with signal from IoT devices. One feasible solution is to perform breathing detection as the replacement of occupancy detection. Chest movements, during inhalation and exhalation, can be potentially used as a proxy measure for detection of breathing and has been demonstrated for short distances [24]. However, breathing detection that can cover the entire area of the flat and under TTW scenario remains a challenge for the current system. This will require a highlevel of sensitivity to detect micro-Doppler variations at a considerable distance and also need to deal with surrounding static background objects.

\section{Multi-Person Activity Recognition}

While we have tested activity recognition for a single user, the more interesting and also challenging problem is the case where multiple people are in the environment. Considering the walking trajectories shown in Fig 16(b), it can be considered as a combination of Doppler spectrograms from two people. A separation process is needed to achieve activity recognition for multi-person. One solution is to use multiple receivers to separate the signals due to two distinct mobile objects with the concepts of MIMO radar. This might also help to distinguish the occupancy of people in different room. Our PIoTR can be easily extended to support the MIMO function by deploying multiple SDR devices for more receiving channels. For now, we leave multi-person recognition as the future work.

\section{CONCLUSIONS}

In this paper, we present PIoTR, a passive IoT-based RF sensing system for occupancy detection and activity recognition. PIoTR is designed with passive radar technique which is not specific to a particular communication protocol. As a result, it can work with different signal sources like WiFi and EH. Unlike other CSI works which use off-the-shelf network cards, we evaluate PIoTR with real transmissions from commercial IoT devices. Experimental results show that, with low frame rate, PIoTR can be used for occupancy detection with 
an average accuracy of $90 \%$. Whilst, with a high frame rate, PIoTR can achieve $91.3 \%$ accuracy in activity recognition and more than $99 \%$ in occupancy detection.

Potential works include the study of the feasibility of other short-range signal sources like NFC, Bluetooth. They could be used for near-field sensing like finger gesture and vital sign detection. Also, studies on extended topics like multi-person recognition, distinguishing animals and humans and control of computational complexity are essential to further deploy PIoTR as a practical system.

\section{REFERENCES}

[1] X. Zhou, W. Liang, I. Kevin, K. Wang, H. Wang, L. T. Yang, and Q. Jin, "Deep-learning-enhanced human activity recognition for internet of healthcare things," IEEE Internet of Things Journal, vol. 7, no. 7, pp. 6429-6438, 2020.

[2] T. Liu, J. Wang, B. Yang, and X. Wang, "Ngdnet: Nonuniform gaussianlabel distribution learning for infrared head pose estimation and on-task behavior understanding in the classroom," Neurocomputing, vol. 436, pp. $210-220,2021$.

[3] M. Nardo, D. Forino, and T. Murino, "The evolution of man-machine interaction: The role of human in industry 4.0 paradigm," Production \& Manufacturing Research, vol. 8, no. 1, pp. 20-34, 2020.

[4] L. Chen and C. D. Nugent, "Sensor-based activity recognition review," in Human Activity Recognition and Behaviour Analysis. Springer, 2019, pp. 23-47.

[5] D. R. Beddiar, B. Nini, M. Sabokrou, and A. Hadid, "Vision-based human activity recognition: a survey," Multimedia Tools and Applications, vol. 79, no. 41, pp. 30 509-30 555, 2020.

[6] M. Muzammal, R. Talat, A. H. Sodhro, and S. Pirbhulal, "A multisensor data fusion enabled ensemble approach for medical data from body sensor networks," Information Fusion, vol. 53, pp. 155-164, 2020.

[7] T. Liu, J. Wang, B. Yang, and X. Wang, "Ngdnet: Nonuniform gaussianlabel distribution learning for infrared head pose estimation and on-task behavior understanding in the classroom," Neurocomputing, vol. 436, pp. 210-220, 2021.

[8] Y. Ma, G. Zhou, and S. Wang, "Wifi sensing with channel state information: A survey," ACM Computing Surveys (CSUR), vol. 52, no. 3, p. 46, 2019.

[9] L. Chettri and R. Bera, "A comprehensive survey on internet of things (iot) toward $5 \mathrm{~g}$ wireless systems," IEEE Internet of Things Journal, vol. 7, no. 1, pp. 16-32, 2019.

[10] A. H. Sodhro, S. Pirbhulal, A. K. Sangaiah, S. Lohano, G. H. Sodhro, and Z. Luo, "5g-based transmission power control mechanism in fog computing for internet of things devices," Sustainability, vol. 10, no. 4, p. 1258,2018

[11] R. v. Nee and R. Prasad, OFDM for wireless multimedia communications. Artech House, Inc., 2000.

[12] X. Wu, Z. Chu, P. Yang, C. Xiang, X. Zheng, and W. Huang, "Twsee: Human activity recognition through the wall with commodity wi-fi devices," IEEE Transactions on Vehicular Technology, vol. 68, no. 1 , pp. 306-319, 2018.

[13] M. Abbas, M. Elhamshary, H. Rizk, M. Torki, and M. Youssef, "Wideep: Wifi-based accurate and robust indoor localization system using deep learning," in 2019 IEEE International Conference on Pervasive Computing and Communications (PerCom. IEEE, 2019, pp. 1-10.

[14] H. Zou, Y. Zhou, J. Yang, and C. J. Spanos, "Device-free occupancy detection and crowd counting in smart buildings with wifi-enabled iot," Energy and Buildings, vol. 174, pp. 309-322, 2018.

[15] "Linux 802.11n CSI tool," https://dhalperi.github.io/linux-80211ncsitool/, (Accessed on 05/11/2020).

[16] S. Al-Sarawi, M. Anbar, K. Alieyan, and M. Alzubaidi, "Internet of things (iot) communication protocols," in 2017 8th International conference on information technology (ICIT). IEEE, 2017, pp. 685-690.

[17] F. Wang, J. Feng, Y. Zhao, X. Zhang, S. Zhang, and J. Han, "Joint activity recognition and indoor localization with wifi fingerprints," IEEE Access, vol. 7, pp. 80058-80 068, 2019.

[18] H. Kuschel, D. Cristallini, and K. E. Olsen, "Tutorial: Passive radar tutorial," IEEE Aerospace and Electronic Systems Magazine, vol. 34, no. 2, pp. 2-19, 2019.
[19] O. Cabrera, C. Bongioanni, F. Filippini, O. Sarabakha, F. Colone, and P. Lombardo, "Detecting drones and human beings with dvb-s based cots passive radar for short-range surveillance," in 2020 IEEE International Radar Conference (RADAR). IEEE, 2020, pp. 37-42.

[20] A. Guillén-Pérez and M. D. C. Baños, "A wifi-based method to count and locate pedestrians in urban traffic scenarios," in 2018 14th International Conference on Wireless and Mobile Computing, Networking and Communications (WiMob). IEEE, 2018, pp. 123-130.

[21] W. Li, B. Tan, and R. J. Piechocki, "Wifi-based passive sensing system for human presence and activity event classification," IET Wireless Sensor Systems, vol. 8, no. 6, pp. 276-283, 2018.

[22] W. Li, R. J. Piechocki, K. Woodbridge, and K. Chetty, "Physical activity sensing via stand-alone wifi device," in 2019 IEEE Global Communications Conference (GLOBECOM). IEEE, 2019, pp. 1-6.

[23] J. Zhang, F. Wu, B. Wei, Q. Zhang, H. Huang, S. W. Shah, and J. Cheng, "Data augmentation and dense-lstm for human activity recognition using wifi signal," IEEE Internet of Things Journal, vol. 8, no. 6, pp. 46284641, 2020.

[24] W. Li, B. Tan, and R. Piechocki, "Passive radar for opportunistic monitoring in e-health applications," IEEE journal of translational engineering in health and medicine, vol. 6, pp. 1-10, 2018.

[25] W. Wang, A. X. Liu, and M. Shahzad, "Gait recognition using wifi signals," in Proceedings of the 2016 ACM International Joint Conference on Pervasive and Ubiquitous Computing. ACM, 2016, pp. 363-373.

[26] W. Wang, A. X. Liu, M. Shahzad, K. Ling, and S. Lu, "Devicefree human activity recognition using commercial wifi devices," IEEE Journal on Selected Areas in Communications, vol. 35, no. 5, pp. 11181131, 2017.

[27] H. Wang, D. Zhang, Y. Wang, J. Ma, Y. Wang, and S. Li, "Rt-fall: A real-time and contactless fall detection system with commodity wifi devices," IEEE Transactions on Mobile Computing, vol. 16, no. 2, pp. 511-526, 2016.

[28] H. Wang, D. Zhang, J. Ma, Y. Wang, Y. Wang, D. Wu, T. Gu, and B. Xie, "Human respiration detection with commodity wifi devices: do user location and body orientation matter?" in Proceedings of the 2016 ACM International Joint Conference on Pervasive and Ubiquitous Computing. ACM, 2016, pp. 25-36.

[29] J. Yang, H. Zou, H. Jiang, and L. Xie, "Device-free occupant activity sensing using wifi-enabled iot devices for smart homes," IEEE Internet of Things Journal, vol. 5, no. 5, pp. 3991-4002, 2018.

[30] K. Chetty, G. E. Smith, and K. Woodbridge, "Through-the-wall sensing of personnel using passive bistatic wifi radar at standoff distances," IEEE Transactions on Geoscience and Remote Sensing, vol. 50, no. 4, pp. 1218-1226, 2011.

[31] B. Tan, K. Woodbridge, and K. Chetty, "A real-time high resolution passive wifi doppler-radar and its applications," in 2014 International Radar Conference. IEEE, 2014, pp. 1-6.

[32] R. Faragher and R. Harle, "Location fingerprinting with bluetooth low energy beacons," IEEE journal on Selected Areas in Communications, vol. 33, no. 11, pp. 2418-2428, 2015.

[33] M. Min, L. Xiao, Y. Chen, P. Cheng, D. Wu, and W. Zhuang, "Learningbased computation offloading for iot devices with energy harvesting," IEEE Transactions on Vehicular Technology, vol. 68, no. 2, pp. 19301941, 2019.

[34] Y. He, Y. Chen, Y. Hu, and B. Zeng, "Wifi vision: Sensing, recognition, and detection with commodity mimo-ofdm wifi," IEEE Internet of Things Journal, vol. 7, no. 9, pp. 8296-8317, 2020.

[35] Powercast energy harvesing transmitter. [Online]. Available: http://www.powercastco.com/products/powercaster-transmitter

[36] $\mathrm{Ni}$ usrp $2921 . \quad$ [Online]. $\quad$ Available: http://sine.ni.com/nips/cds/view/p/lang/en/nid/212995

[37] K. Greff, R. K. Srivastava, J. Koutník, B. R. Steunebrink, and J. Schmidhuber, "Lstm: A search space odyssey," IEEE transactions on neural networks and learning systems, vol. 28, no. 10, pp. 2222-2232, 2016.

[38] Z. Zeng, M. G. Amin, and T. Shan, "Arm motion classification using time-series analysis of the spectrogram frequency envelopes," Remote Sensing, vol. 12, no. 3, p. 454, 2020.

[39] J. J. Lehtomaki, J. Vartiainen, M. Juntti, and H. Saarnisaari, "Cfar outlier detection with forward methods," IEEE Transactions on Signal Processing, vol. 55, no. 9, pp. 4702-4706, 2007.

[40] K. Simonyan and A. Zisserman, "Very deep convolutional networks for large-scale image recognition," arXiv preprint arXiv:1409.1556, 2014. 